\title{
Changes In UNDERGRADUATE AND GRADUATE STUDENT PERSPECTIVES OF ONLINE VERSUS FACE-TO-FACE EDUCATION
}

\author{
Lynn A. Fish and Coral R. Snodgrass
}

The BRC Academy Journal of Business 11, no. 1 (2021): 143-161.

https://dx.doi.org/10.15239/j.brcacadjb.2020.11.01.ja06

\section{WEB APPENDIX}

https://dx.doi.org/10.15239/j.brcacadjb.2021.11.01.wa06 
Web Appendix

Table 1. Number of Undergraduate and Graduate Students Online and FTF in 2012 \& 2018

\begin{tabular}{|c|c|c|c|c|c|c|c|c|}
\hline $\begin{array}{c}\# \text { of } \\
\text { Students }\end{array}$ & \multicolumn{3}{|c|}{ Online } & \multicolumn{3}{c|}{ FTF } & \multicolumn{2}{c|}{ Total } \\
\cline { 2 - 9 } & Undergraduate & Graduate & Total & Undergraduate & Graduate & Total & UG & G \\
\hline 2012 & 22 & 22 & 44 & 41 & 25 & 66 & 63 & 47 \\
\hline 2018 & 40 & 42 & 82 & 34 & 18 & 52 & 74 & 60 \\
\hline Total & 62 & 64 & 126 & 75 & 43 & 118 & 137 & 107 \\
\hline
\end{tabular}

Table 2. 2012 OL Undergraduate and Graduate Student Results (Fish \& Snodgrass, 2014)

\begin{tabular}{|l|c|c|c|c|c|c|c|c|c|c|c|c|c|}
\hline \multirow{2}{*}{ Metric } & \multicolumn{7}{|c|}{ \# of Undergraduate Responses } & \multicolumn{5}{c|}{ \# of Graduate Responses } \\
\cline { 2 - 21 } & 1 & 2 & 3 & 4 & 5 & 1 & 2 & 3 & 4 & 5 \\
\hline Difficulty & 5 & 8 & 6 & 3 & 0 & 2 & 9 & 8 & 3 & 0 \\
\hline Motivation & 4 & 5 & 11 & 2 & 0 & 6 & 6 & 10 & 0 & 0 \\
\hline Student-to-student Interaction & 0 & 8 & 10 & 4 & 0 & 2 & 13 & 3 & 3 & 1 \\
\hline Instructor-to-student Interaction & 2 & 8 & 5 & 6 & 1 & 2 & 12 & 5 & 3 & 0 \\
\hline Discipline & 3 & 4 & 7 & 7 & 1 & 2 & 5 & 3 & 10 & 2 \\
\hline Cheat & 3 & 9 & 6 & 3 & 1 & 3 & 10 & 7 & 2 & 0 \\
\hline Self-directed & 1 & 5 & 8 & 5 & 3 & 3 & 7 & 8 & 4 & 0 \\
\hline Independence & 1 & 2 & 7 & 8 & 4 & 1 & 5 & 2 & 10 & 4 \\
\hline Schedule flexibility & 0 & 0 & 3 & 7 & 12 & 0 & 3 & 2 & 9 & 8 \\
\hline Time investment & 0 & 8 & 7 & 7 & 0 & 2 & 6 & 8 & 6 & 0 \\
\hline Cost investment & 0 & 7 & 14 & 1 & 0 & 1 & 7 & 12 & 2 & 0 \\
\hline Preference opposite? & 9 & 11 & 2 & & & 12 & 7 & 3 & & \\
\hline Happiness with environment & 1 & 0 & 10 & 7 & 4 & 2 & 3 & 9 & 6 & 2 \\
\hline Appropriateness of Online & 15 & 6 & 1 & & & 11 & 4 & 7 & & \\
\hline
\end{tabular}


Table 3. Chi-Square Analysis 2012 OL Undergraduate vs Graduate Perspectives

\begin{tabular}{|c|c|c|c|c|c|}
\hline Metric & $\begin{array}{c}\text { Pearson } \\
\text { Chi-Square } \\
\text { Value }\end{array}$ & Df & $\begin{array}{c}\text { Asymptotic } \\
\text { Significance } \\
(2 \text {-sided })\end{array}$ & $\begin{array}{c}\text { Pearson's } \\
\mathrm{R}\end{array}$ & $\begin{array}{c}\text { Spearman } \\
\text { Correlation }\end{array}$ \\
\hline Difficulty & 1.630 & 3 & .653 & .124 & .126 \\
\hline Motivation & 2.539 & 3 & .468 & -.181 & -.177 \\
\hline Student-to-student Interaction & 8.103 & 4 & $.088 * *$ & -.206 & -.266 \\
\hline Student-to-instructor Interact & 2.800 & 4 & .592 & -.208 & -.196 \\
\hline Discipline & 2.774 & 4 & .596 & .119 & .132 \\
\hline Cheat & 1.330 & 4 & .856 & -.907 & -.070 \\
\hline Self-directed & 4.444 & 4 & .349 & -.282 & -.261 \\
\hline Independence & 4.286 & 4 & .369 & -.021 & .006 \\
\hline Schedule flexibility & 4.250 & 3 & .236 & -.229 & -.203 \\
\hline Time investment & 2.429 & 3 & .488 & -.077 & -.057 \\
\hline Cost investment & 1.487 & 3 & .685 & -.036 & -.027 \\
\hline Preference opposite? & 1.517 & 2 & .468 & -.067 & -.093 \\
\hline Happiness with environment & 4.160 & 4 & .389 & -.222 & -.216 \\
\hline Appropriateness of Online & 5.515 & 2 & $.063 * *$ & .292 & .256 \\
\hline
\end{tabular}

$* \mathrm{p} \leq .05, * * \mathrm{p} \leq .10$

Table 4. 2012 FTF Undergraduate and Graduate Student Results (Fish \& Snodgrass, 2014)

\begin{tabular}{|l|c|c|c|c|c|c|c|c|c|c|c|c|}
\hline \multirow{2}{*}{ Metric } & \multicolumn{7}{|c|}{ \# of Undergraduate Responses } & \multicolumn{6}{|c|}{ \# of Graduate Responses } \\
\cline { 2 - 15 } & 1 & 2 & 3 & 4 & 5 & 1 & 2 & 3 & 4 & 5 \\
\hline Difficulty & 4 & 22 & 4 & 10 & 1 & 1 & 9 & 6 & 9 & 0 \\
\hline Motivation & 0 & 1 & 7 & 16 & 17 & 0 & 1 & 7 & 12 & 5 \\
\hline Student-to-student Interaction & 0 & 0 & 5 & 21 & 15 & 1 & 1 & 1 & 11 & 11 \\
\hline Student-to-instructor Interact & 0 & 0 & 2 & 19 & 20 & 1 & 0 & 1 & 10 & 13 \\
\hline Discipline & 1 & 9 & 9 & 13 & 9 & 0 & 8 & 6 & 8 & 3 \\
\hline Cheat & 1 & 1 & 5 & 25 & 9 & 0 & 0 & 9 & 10 & 6 \\
\hline Self-directed & 0 & 2 & 5 & 26 & 8 & 0 & 0 & 5 & 15 & 5 \\
\hline Independence & 0 & 5 & 15 & 19 & 2 & 0 & 3 & 11 & 10 & 1 \\
\hline Schedule flexibility & 6 & 15 & 8 & 10 & 2 & 1 & 12 & 7 & 4 & 1 \\
\hline Time investment & 0 & 6 & 14 & 19 & 1 & 1 & 1 & 10 & 12 & 1 \\
\hline Cost investment & 1 & 4 & 16 & 17 & 3 & 0 & 3 & 7 & 12 & 2 \\
\hline Preference opposite? & 3 & 12 & 26 & & & 2 & 11 & 11 & & \\
\hline Happiness with environment & 0 & 0 & 6 & 18 & 17 & 0 & 0 & 5 & 11 & 9 \\
\hline Appropriateness of Online & 16 & 18 & 7 & & & 11 & 9 & 5 & & \\
\hline
\end{tabular}

Table 5. Chi-Square Analysis 2012 FTF Undergraduate vs Graduate Perspectives 


\begin{tabular}{|c|c|c|c|c|c|}
\hline Metric & $\begin{array}{c}\text { Pearson } \\
\text { Chi-Square } \\
\text { Value }\end{array}$ & Df & $\begin{array}{c}\text { Asymptotic } \\
\text { Significance } \\
\text { (2-sided) }\end{array}$ & $\begin{array}{c}\text { Pearson's } \\
\mathrm{R}\end{array}$ & $\begin{array}{c}\text { Spearman } \\
\text { Correlation }\end{array}$ \\
\hline Difficulty & 5.127 & 4 & .275 & .172 & .189 \\
\hline Motivation & 3.440 & 3 & .329 & -.212 & -.221 \\
\hline Student-to-student Interaction & 4.811 & 4 & .307 & -.027 & .052 \\
\hline Student-to-instructor Interaction & 1.841 & 3 & .606 & -.053 & .012 \\
\hline Discipline & 2.094 & 4 & .719 & -.109 & -.115 \\
\hline Cheat & 6.686 & 4 & .153 & -.053 & -.104 \\
\hline Self-directed & 1.875 & 3 & .599 & .017 & -.009 \\
\hline Independence & .386 & 3 & .943 & -.051 & -.058 \\
\hline Schedule flexibility & 3.185 & 4 & .527 & -.001 & .006 \\
\hline Time investment & 3.546 & 4 & .471 & .041 & .049 \\
\hline Cost investment & 1.375 & 4 & .849 & .073 & .075 \\
\hline Preference opposite? & 2.016 & 2 & .365 & -.141 & -.159 \\
\hline Happiness with environment & .386 & 2 & .824 & -.074 & -.071 \\
\hline Appropriateness of Online & .404 & 2 & .817 & -.014 & -.021 \\
\hline
\end{tabular}

$* \mathrm{p} \leq .05,{ }^{* *} \mathrm{p} \leq .10$

Table 6. 2018 OL Undergraduate and Graduate Student Results

\begin{tabular}{|l|c|c|c|c|c|c|c|c|c|c|c|}
\hline \multirow{2}{*}{ Metric } & \multicolumn{9}{|c|}{ \# of Undergraduate Responses } & \multicolumn{3}{|c|}{ \# of Graduate Responses } \\
\cline { 2 - 18 } & 1 & 2 & 3 & 4 & 5 & 1 & 2 & 3 & 4 & 5 \\
\hline Difficulty & 6 & 14 & 17 & 3 & 0 & 2 & 15 & 18 & 7 & 0 \\
\hline Motivation & 4 & 10 & 16 & 9 & 1 & 6 & 12 & 17 & 6 & 0 \\
\hline Student-to-student Interaction & 4 & 10 & 13 & 10 & 3 & 9 & 19 & 9 & 5 & 0 \\
\hline Instructor-to-student Interaction & 2 & 15 & 20 & 3 & 0 & 3 & 18 & 14 & 4 & 2 \\
\hline Discipline & 2 & 6 & 13 & 13 & 6 & 2 & 5 & 17 & 15 & 3 \\
\hline Cheat & 5 & 16 & 17 & 2 & 0 & 10 & 11 & 20 & 1 & 0 \\
\hline Self-directed & 0 & 5 & 8 & 16 & 11 & 4 & 5 & 14 & 13 & 6 \\
\hline Independence & 0 & 3 & 5 & 20 & 12 & 3 & 3 & 11 & 16 & 9 \\
\hline Schedule flexibility & 0 & 0 & 2 & 15 & 23 & 1 & 0 & 6 & 17 & 18 \\
\hline Time investment & 2 & 7 & 27 & 4 & 0 & 2 & 5 & 21 & 11 & 3 \\
\hline Cost investment & 0 & 10 & 25 & 5 & 0 & 3 & 8 & 26 & 5 & 0 \\
\hline Preference opposite? & 16 & 18 & 6 & & & 19 & 15 & 8 & & \\
\hline Happiness with environment & 0 & 1 & 10 & 19 & 10 & 3 & 5 & 14 & 10 & 10 \\
\hline Appropriateness of Online & 34 & 6 & 0 & & & 33 & 6 & 3 & & \\
\hline
\end{tabular}

Table 7. Chi-Square Analysis 2018 OL Undergraduate vs Graduate Perspectives 


\begin{tabular}{|c|c|c|c|c|c|}
\hline Metric & $\begin{array}{c}\text { Pearson } \\
\text { Chi-Square } \\
\text { Value }\end{array}$ & $\begin{array}{c}\mathrm{D} \\
\mathrm{f}\end{array}$ & $\begin{array}{c}\text { Asymptotic } \\
\text { Significance } \\
(2-\text {-sided })\end{array}$ & $\begin{array}{c}\text { Pearson's } \\
\mathrm{R}\end{array}$ & $\begin{array}{c}\text { Spearman } \\
\text { Correlation }\end{array}$ \\
\hline Difficulty & 3.616 & 3 & .306 & .175 & .158 \\
\hline Motivation & 2.200 & 4 & .699 & -.139 & -.132 \\
\hline Student-to-student Interaction & 10.067 & 4 & $.039 *$ & -.333 & -.328 \\
\hline Student-to-instructor Interact & 3.985 & 4 & .408 & .026 & -.017 \\
\hline Discipline & 1.719 & 4 & .787 & -.043 & -.047 \\
\hline Cheat & 3.122 & 3 & .373 & -.070 & -.048 \\
\hline Self-directed & 7.373 & 4 & .117 & -.246 & -.237 \\
\hline Independence & 6.078 & 4 & .193 & -.211 & -.198 \\
\hline Schedule flexibility & 3.688 & 3 & .297 & -.205 & -.192 \\
\hline Time investment & 7.306 & 4 & .121 & .223 & .232 \\
\hline Cost investment & 3.195 & 3 & .363 & -.066 & -.033 \\
\hline Preference opposite? & .767 & 2 & .681 & -.008 & -.019 \\
\hline Happiness with environment & 9.083 & 4 & $.059 * *$ & -.241 & -.214 \\
\hline Appropriateness of Online & 2.968 & 2 & .227 & .137 & .097 \\
\hline
\end{tabular}

$* \mathrm{p} \leq .05, * * \mathrm{p} \leq .10$

Table 8. 2018 FTF Undergraduate and Graduate Student Results

\begin{tabular}{|l|c|c|c|c|c|c|c|c|c|c|c|c|}
\hline \multirow{2}{*}{ Metric } & \multicolumn{7}{|c|}{ \# of Undergraduate Responses } & \multicolumn{6}{c|}{ \# of Graduate Responses } \\
\cline { 2 - 17 } & 1 & 2 & 3 & 4 & 5 & 1 & 2 & 3 & 4 & 5 \\
\hline Difficulty & 1 & 14 & 7 & 12 & 0 & 1 & 4 & 7 & 5 & 1 \\
\hline Motivation & 0 & 2 & 8 & 13 & 11 & 1 & 3 & 5 & 4 & 5 \\
\hline Student-to-student Interaction & 0 & 2 & 6 & 14 & 12 & 2 & 0 & 5 & 6 & 5 \\
\hline Instructor-to-student Interaction & 0 & 0 & 8 & 14 & 12 & 2 & 0 & 5 & 6 & 5 \\
\hline Discipline & 0 & 6 & 9 & 11 & 8 & 0 & 5 & 5 & 6 & 2 \\
\hline Cheat & 0 & 3 & 6 & 15 & 10 & 1 & 0 & 4 & 11 & 2 \\
\hline Self-directed & 1 & 7 & 8 & 12 & 6 & 2 & 2 & 5 & 7 & 2 \\
\hline Independence & 0 & 7 & 11 & 13 & 2 & 2 & 3 & 6 & 6 & 1 \\
\hline Schedule flexibility & 7 & 10 & 8 & 8 & 0 & 2 & 9 & 3 & 3 & 0 \\
\hline Time investment & 1 & 5 & 12 & 11 & 5 & 0 & 2 & 8 & 6 & 2 \\
\hline Cost investment & 0 & 4 & 12 & 12 & 6 & 1 & 1 & 8 & 5 & 3 \\
\hline Preference opposite? & 9 & 10 & 13 & & & 6 & 6 & 6 & & \\
\hline Happiness with environment & 0 & 0 & 14 & 10 & 9 & 2 & 1 & 3 & 9 & 3 \\
\hline Appropriateness of Online & 20 & 7 & 6 & & & 12 & 5 & 1 & & \\
\hline
\end{tabular}

Table 9. Chi-Square Analysis 2018 FTF Undergraduate vs Graduate Perspectives 


\begin{tabular}{|c|c|c|c|c|c|}
\hline Metric & $\begin{array}{c}\text { Pearson } \\
\text { Chi-Square } \\
\text { Value }\end{array}$ & Df & $\begin{array}{c}\text { Asymptotic } \\
\text { Significanc } \\
\text { e (2-sided) }\end{array}$ & $\begin{array}{c}\text { Pearson's } \\
\text { R }\end{array}$ & $\begin{array}{c}\text { Spearman } \\
\text { Correlation }\end{array}$ \\
\hline Difficulty & 4.987 & 4 & .289 & .087 & .085 \\
\hline Motivation & 4.401 & 4 & .355 & -.216 & -.181 \\
\hline Student-to-student Interaction & 5.799 & 4 & .215 & -.184 & -.145 \\
\hline Student-to-instructor Interaction & 4.254 & 3 & .235 & -.223 & -.160 \\
\hline Discipline & 1.526 & 3 & .676 & -.157 & -.155 \\
\hline Cheat & 5.993 & 4 & .200 & -.116 & -.124 \\
\hline Self-directed & 2.426 & 4 & .658 & -.070 & -.056 \\
\hline Independence & 3.909 & 4 & .418 & -.124 & -.095 \\
\hline Schedule flexibility & 2.513 & 3 & .473 & -.048 & -.047 \\
\hline Time investment & 1.015 & 4 & .908 & .016 & .003 \\
\hline Cost investment & 2.827 & 4 & .587 & -.072 & -.060 \\
\hline Preference opposite? & .281 & 2 & .869 & -.073 & -.074 \\
\hline Happiness with environment & 9.588 & 4 & $.048 *$ & -.145 & -.057 \\
\hline Appropriateness of Online & 1.634 & 2 & .442 & -.123 & -.096 \\
\hline
\end{tabular}

$* \mathrm{p} \leq .05,{ }^{* *} \mathrm{p} \leq .10$

Table 10. 2012 Undergraduate Students OL versus FTF

\begin{tabular}{|c|c|c|c|c|c|}
\hline Metric & $\begin{array}{c}\text { Pearson } \\
\text { Chi-Square } \\
\text { Value }\end{array}$ & Df & $\begin{array}{c}\text { Asymptotic } \\
\text { Significance } \\
\text { (2-sided) }\end{array}$ & $\begin{array}{c}\text { Pearson's } \\
\mathrm{R}\end{array}$ & $\begin{array}{c}\text { Spearman } \\
\text { Correlation }\end{array}$ \\
\hline Difficulty & 6.849 & 4 & .144 & .122 & .094 \\
\hline Motivation & 30.617 & 4 & $.000^{*}$ & .686 & .681 \\
\hline Student-to-student Interaction & 31.167 & 3 & $.000^{*}$ & .694 & .679 \\
\hline Student-to-instructor Interact & 29.935 & 4 & $.000^{*}$ & .684 & .644 \\
\hline Discipline & 8.047 & 4 & $.090^{* *}$ & .261 & .248 \\
\hline Cheat & 25.980 & 4 & $.000^{*}$ & .595 & .586 \\
\hline Self-directed & 12.352 & 3 & $.006^{*}$ & .360 & .349 \\
\hline Independence & 3.473 & 3 & .324 & -.149 & -.140 \\
\hline Schedule flexibility & 28.944 & 4 & $.000^{*}$ & -.646 & -.659 \\
\hline Time investment & 3.942 & 3 & .268 & .249 & .242 \\
\hline Cost investment & 13.277 & 4 & $.010^{*}$ & .378 & .413 \\
\hline Preference opposite? & 17.000 & 2 & $.000^{*}$ & .520 & .527 \\
\hline Happiness with environment & 9.541 & 2 & $.008^{*}$ & .379 & .376 \\
\hline Appropriateness of Online & 5.391 & 2 & $.068^{* *}$ & .288 & .296 \\
\hline
\end{tabular}

Table 11. 2018 Undergraduate Students OL versus FTF 


\begin{tabular}{|c|c|c|c|c|c|}
\hline Metric & $\begin{array}{c}\text { Pearson } \\
\text { Chi-Square } \\
\text { Value }\end{array}$ & Df & $\begin{array}{c}\text { Asymptotic } \\
\text { Significance } \\
(2 \text {-sided })\end{array}$ & $\begin{array}{c}\text { Pearson's } \\
\text { R }\end{array}$ & $\begin{array}{c}\text { Spearman } \\
\text { Correlation }\end{array}$ \\
\hline Difficulty & 12.735 & 3 & $.005^{*}$ & .251 & .222 \\
\hline Motivation & 20.710 & 4 & $.000^{*}$ & .521 & .522 \\
\hline Student-to-student Interaction & 17.608 & 4 & $.001^{*}$ & .484 & .487 \\
\hline Student-to-instructor Interact & 41.044 & 4 & $.000^{*}$ & .721 & .734 \\
\hline Discipline & 2.711 & 4 & .607 & .115 & .104 \\
\hline Cheat & 38.866 & 4 & $.000^{*}$ & .677 & .683 \\
\hline Self-directed & 2.908 & 4 & .573 & -.183 & -.174 \\
\hline Independence & 11.916 & 3 & $.008^{*}$ & -.386 & -.398 \\
\hline Schedule flexibility & 45.477 & 4 & $.000^{*}$ & -.764 & -.780 \\
\hline Time investment & 14.310 & 4 & $.006^{*}$ & .329 & .327 \\
\hline Cost investment & 15.638 & 3 & $.001^{*}$ & .424 & .412 \\
\hline Preference opposite? & 6.010 & 2 & $.050^{*}$ & .240 & .234 \\
\hline Happiness with environment & 3.877 & 3 & .275 & -.063 & -.078 \\
\hline Appropriateness of Online & 9.119 & 2 & $.010^{*}$ & .339 & .305 \\
\hline
\end{tabular}

$* \mathrm{p} \leq .05,{ }^{* *} \mathrm{p} \leq .10$

Table 12. Chi-Square Analysis Undergraduate OL Students 2012 vs 2018

\begin{tabular}{|c|c|c|c|c|c|}
\hline Metric & $\begin{array}{c}\text { Pearson Chi- } \\
\text { Square Value }\end{array}$ & Df & $\begin{array}{c}\text { Asymptotic } \\
\text { Significance } \\
(2 \text {-sided) }\end{array}$ & $\begin{array}{c}\text { Pearson's } \\
\mathrm{R}\end{array}$ & $\begin{array}{c}\text { Spearman } \\
\text { Correlation }\end{array}$ \\
\hline Difficulty & 1.925 & 3 & .588 & .058 & .069 \\
\hline Motivation & 3.533 & 4 & .544 & .163 & .151 \\
\hline Student-to-student Interact & 5.416 & 4 & .247 & .064 & .069 \\
\hline Student-to-instructor Interact & 8.632 & 4 & $.071^{* *}$ & -.122 & -.088 \\
\hline Discipline & 2.780 & 4 & .595 & .183 & .168 \\
\hline Cheat & 4.035 & 4 & .401 & -.080 & -.037 \\
\hline Self-directed & 6.670 & 4 & .154 & .292 & .287 \\
\hline Independence & 5.952 & 4 & .203 & .242 & .238 \\
\hline Schedule flexibility & 1.464 & 2 & .481 & .087 & .061 \\
\hline Time investment & 10.291 & 3 & $.016^{*}$ & -.085 & -.057 \\
\hline Cost investment & 1.172 & 2 & .557 & .121 & .116 \\
\hline Preference opposite? & .463 & 2 & .793 & .048 & .039 \\
\hline Happiness with environment & 5.334 & 4 & .255 & .202 & .194 \\
\hline Appropriateness of Online & 3.431 & 2 & .180 & -.225 & -.205 \\
\hline
\end{tabular}

$* \mathrm{p} \leq .05,{ }^{* *} \mathrm{p} \leq .10$ 
Table 13. Chi-Square Analysis Undergraduate FTF Students 2012 vs 2018

\begin{tabular}{|c|c|c|c|c|c|}
\hline Metric & $\begin{array}{c}\text { Pearson } \\
\text { Chi-Square } \\
\text { Value }\end{array}$ & Df & $\begin{array}{c}\text { Asymptotic } \\
\text { Significance } \\
\text { (2-sided) }\end{array}$ & $\begin{array}{c}\text { Pearson's } \\
\mathrm{R}\end{array}$ & $\begin{array}{c}\text { Spearman } \\
\text { Correlation }\end{array}$ \\
\hline Difficulty & 4.968 & 4 & .291 & .159 & .175 \\
\hline Motivation & 1.355 & 3 & .716 & -.131 & -.124 \\
\hline Student-to-student Interaction & 3.199 & 3 & .362 & -.120 & -.085 \\
\hline Student-to-instructor Interact & 5.754 & 2 & $.056^{* *}$ & -.232 & -.211 \\
\hline Discipline & 1.182 & 4 & .881 & .059 & .050 \\
\hline Cheat & 4.025 & 4 & .403 & -.020 & -.011 \\
\hline Self-directed & 9.342 & 4 & $.053^{* *}$ & -.282 & -.257 \\
\hline Independence & 1.223 & 3 & .747 & -.083 & -.079 \\
\hline Schedule flexibility & 2.463 & 4 & .651 & -.075 & -.064 \\
\hline Time investment & 5.595 & 4 & .231 & .021 & .018 \\
\hline Cost investment & 2.805 & 4 & .591 & .098 & .081 \\
\hline Preference opposite? & 6.504 & 2 & $.039^{*}$ & -.289 & -.273 \\
\hline Happiness with environment & 7.166 & 2 & $.028^{*}$ & -.266 & -.260 \\
\hline Appropriateness of Online & 4.550 & 2 & .103 & -.135 & -.162 \\
\hline
\end{tabular}

$* \mathrm{p} \leq .05, * * \mathrm{p} \leq .10$

Table 14. 2012 Graduate Students OL versus FTF

\begin{tabular}{|c|c|c|c|c|c|}
\hline Metric & $\begin{array}{c}\text { Pearson } \\
\text { Chi- } \\
\text { Square } \\
\text { Value }\end{array}$ & Df & $\begin{array}{c}\text { Asymptotic } \\
\text { Significance } \\
(2 \text {-sided) }\end{array}$ & $\begin{array}{c}\text { Pearson's } \\
\mathrm{R}\end{array}$ & $\begin{array}{c}\text { Spearman } \\
\text { Correlation }\end{array}$ \\
\hline Difficulty & 3.442 & 3 & .328 & .205 & .195 \\
\hline Motivation & 27.019 & 4 & $.000^{*}$ & .716 & .740 \\
\hline Student-to-student Interaction & 24.432 & 4 & $.000^{*}$ & .663 & .658 \\
\hline Student-to-instructor Interaction & 31.707 & 4 & $.000^{*}$ & .748 & .757 \\
\hline Discipline & 3.939 & 4 & .414 & .006 & -.016 \\
\hline Cheat & 24.492 & 4 & $.000^{*}$ & .689 & .702 \\
\hline Self-directed & 21.959 & 4 & $.000^{*}$ & .665 & .676 \\
\hline Independence & 9.377 & 4 & $.052^{*}$ & -.073 & -.125 \\
\hline Schedule flexibility & 16.421 & 4 & $.003^{*}$ & -.565 & -.563 \\
\hline Time investment & 6.964 & 4 & .138 & .337 & .338 \\
\hline Cost investment & 12.996 & 4 & $.011^{*}$ & .491 & .498 \\
\hline Preference opposite? & 12.540 & 2 & $.002^{*}$ & .502 & .502 \\
\hline Happiness with environment & 11.925 & 4 & $.018 *$ & .494 & .488 \\
\hline Appropriateness of Online & 2.073 & 2 & .355 & -.035 & -.017 \\
\hline
\end{tabular}

$* \mathrm{p} \leq .05, * * \mathrm{p} \leq .10$ 
Table 15. 2018 Graduate Students OL versus FTF

\begin{tabular}{|c|c|c|c|c|c|}
\hline Metric & $\begin{array}{c}\text { Pearson } \\
\text { Chi- } \\
\text { Square } \\
\text { Value }\end{array}$ & Df & $\begin{array}{c}\text { Asymptotic } \\
\text { Significance } \\
(2-\text {-sided })\end{array}$ & $\begin{array}{c}\text { Pearson's } \\
\mathrm{R}\end{array}$ & $\begin{array}{c}\text { Spearman } \\
\text { Correlation }\end{array}$ \\
\hline Difficulty & 3.899 & 4 & .420 & .181 & .173 \\
\hline Motivation & 14.092 & 4 & $.007 *$ & .392 & .359 \\
\hline Student-to-student Interaction & 23.915 & 4 & $.000 *$ & .543 & .520 \\
\hline Student-to-instructor Interaction & 16.952 & 4 & $.002 *$ & .415 & .424 \\
\hline Discipline & 3.575 & 4 & .467 & -.004 & -.018 \\
\hline Cheat & 35.433 & 4 & $.000 *$ & .609 & .640 \\
\hline Self-directed & .495 & 4 & .974 & -.003 & .008 \\
\hline Independence & 3.591 & 4 & .464 & -.219 & -.230 \\
\hline Schedule flexibility & 33.567 & 4 & $.000 *$ & -.682 & -.659 \\
\hline Time investment & 1.409 & 4 & .843 & .130 & .120 \\
\hline Cost investment & 11.159 & 4 & $.025 *$ & .341 & .341 \\
\hline Preference opposite? & 1.551 & 2 & .460 & .153 & .148 \\
\hline Happiness with environment & 5.007 & 4 & .287 & .040 & .064 \\
\hline Appropriateness of Online & 1.537 & 2 & .464 & .080 & .112 \\
\hline
\end{tabular}

$* \mathrm{p} \leq .05, * * \mathrm{p} \leq .10$

Table 16. Comparison of OL Graduate Students 2012 vs 2018

\begin{tabular}{|c|c|c|c|c|c|}
\hline Metric & $\begin{array}{c}\text { Pearson } \\
\text { Chi-Square } \\
\text { Value }\end{array}$ & $\begin{array}{c}\mathrm{D} \\
\mathrm{f}\end{array}$ & $\begin{array}{c}\text { Asymptotic } \\
\text { Significance } \\
(2 \text {-sided })\end{array}$ & $\begin{array}{c}\text { Pearson's } \\
\mathrm{R}\end{array}$ & $\begin{array}{c}\text { Spearman } \\
\text { Correlation }\end{array}$ \\
\hline Difficulty & .771 & 3 & .856 & .098 & .097 \\
\hline Motivation & 4.493 & 3 & .213 & .200 & .186 \\
\hline Student-to-student Interaction & 4.244 & 4 & .374 & -.108 & -.087 \\
\hline Student-to-instructor Interaction & 2.120 & 4 & .714 & .121 & .119 \\
\hline Discipline & 5.264 & 4 & .261 & .027 & -.009 \\
\hline Cheat & 4.610 & 3 & .203 & -.044 & -.015 \\
\hline Self-directed & 7.344 & 4 & .119 & .294 & .303 \\
\hline Independence & 5.307 & 4 & .257 & .040 & .028 \\
\hline Schedule flexibility & 6.713 & 4 & .152 & .111 & .091 \\
\hline Time investment & 4.587 & 4 & .332 & .189 & .174 \\
\hline Cost investment & 1.397 & 3 & .706 & .068 & .090 \\
\hline Preference opposite? & .568 & 2 & .753 & .094 & .094 \\
\hline Happiness with environment & 2.073 & 4 & .722 & .131 & .132 \\
\hline Appropriateness of Online & 7.481 & 2 & $.024 *$ & -.337 & -.320 \\
\hline
\end{tabular}

$* \mathrm{p} \leq .05, * * \mathrm{p} \leq .10$ 
Table 17. Comparison of FTF Graduate Students 2012 vs 2018

\begin{tabular}{|c|c|c|c|c|c|}
\hline Metric & $\begin{array}{c}\text { Pearson } \\
\text { Chi- } \\
\text { Square } \\
\text { Value }\end{array}$ & Df & $\begin{array}{c}\text { Asymptotic } \\
\text { Significance } \\
\text { (2-sided) }\end{array}$ & $\begin{array}{c}\text { Pearson's } \\
\mathrm{R}\end{array}$ & $\begin{array}{c}\text { Spearman } \\
\text { Correlation }\end{array}$ \\
\hline Difficulty & 3.085 & 4 & .544 & .070 & .064 \\
\hline Motivation & 5.335 & 4 & .255 & -.168 & -.129 \\
\hline Student-to-student Interaction & 6.760 & 4 & .149 & -.237 & -.255 \\
\hline Student-to-instructor Interaction & 6.591 & 3 & $.086 * *$ & -.315 & -.333 \\
\hline Discipline & .133 & 3 & .988 & .018 & .020 \\
\hline Cheat & 3.935 & 3 & .269 & -.096 & -.037 \\
\hline Self-directed & 7.247 & 4 & .123 & -.374 & -.330 \\
\hline Independence & 3.422 & 4 & .490 & -.165 & -.125 \\
\hline Schedule flexibility & 2.056 & 4 & .726 & -.142 & -.143 \\
\hline Time investment & 2.824 & 4 & .588 & .003 & -.037 \\
\hline Cost investment & 4.381 & 4 & .357 & -.053 & -.057 \\
\hline Preference opposite? & 4.169 & 2 & .124 & -.250 & -.231 \\
\hline Happiness with environment & 5.712 & 4 & .222 & -.303 & -.250 \\
\hline Appropriateness of Online & 2.787 & 2 & .248 & -.254 & -.249 \\
\hline
\end{tabular}

$* \mathrm{p} \leq .05, * * \mathrm{p} \leq .10$

\section{APPENDIX A \\ RESEARCH HYPOTHESES}

\#1) Do undergraduate and graduate business students in 2012 and 2018 perceive the OL and FTF learning environments equally for all individual and program factors?

Hypotheses:

$H_{1 o A}$ : For business students in 2012, undergraduate and graduate students ' perceptions of OL education for individual and program factors are equal to their perceptions of FTF education.

$H_{11 A}$ : For business students in 2012, undergraduate and graduate students ' perceptions of OL education for individual and program factors are not equal to their perceptions of FTF education.

$H_{1 o B}$ : For business students in 2018, undergraduate and graduate students' perceptions of OL education for individual and program factors are equal to their perceptions of FTF education.

$H_{11 B}$ : For business students in 2018, undergraduate and graduate students' perceptions of OL education for individual and program factors are not equal to their perceptions of FTF education.

\#2) Have undergraduate and graduate business students' perspectives on OL and FTF learning environments changed over the past 6 years? 
Hypotheses:

$H_{2 o A}$ : In the OL environment, over the past 6 years, undergraduate and graduate students' perceptions of OL education individual and program factors versus FTF have remained the same.

$H_{21 B}$ : In the OL environment, over the past 6 years, undergraduate and graduate students' perceptions of OL education individual and program factors versus FTF have changed.

$H_{2 o B}$ : In the FTF environment, over the past 6 years, undergraduate and graduate students' perceptions of OL education individual and program factors versus FTF have remained the same.

$H_{21 B}$ : In the FTF environment, over the past 6 years, undergraduate and graduate students' perceptions of OL education individual and program factors versus FTF have changed.

\#3) Do undergraduate students who have and have not experienced OL education, perceive the $O L$ and FTF environments equally for all individual and program factors?

$H_{30} A$ : In the online environment, over the past 6 years, undergraduate students' perceptions of their educational preference in a particular learning environment remain unchanged for all factors.

$H_{31 A}$ : In the online environment, over the past 6 years, undergraduate students' perceptions of their educational preference in a particular learning environment changed for all factors.

$H_{30 B}$ : In the FTF environment, over the past 6 years, undergraduate students' perceptions of their educational preference in a particular learning environment remain unchanged for all factors.

$H_{31 B}$ : In the FTF environment, over the past 6 years, undergraduate students' perceptions of their educational preference in a particular learning environment changed for all factors.

\#4) Do graduate students who have and have not experienced OL education, perceive the OL and FTF environments equally for all individual and program factors?

$H_{40 \mathrm{~A}}$ : In the online environment, over the past 6 years, graduate students' perceptions of their educational preference in a particular learning environment remain unchanged for all factors.

$H_{4 A 1}$ : In the online environment, over the past 6 years, graduate students' perceptions of their educational preference in a particular learning environment changed for all factors.

$H_{40 B}$ : In the FTF environment, over the past 6 years, graduate students' perceptions of cheating remain unchanged for all factors.

$H_{41 B}$ : In the FTF environment, over the past 6 years, graduate students' perceptions of cheating changed for all factors. 


\section{APPENDIX B \\ Student Perceptions Survey}

With the rise in online education, we are interested in gathering information as to student perceptions of educational methods. We would greatly appreciate you completing this anonymous form. Thank you!

\section{Background Information:}

\begin{tabular}{|c|c|}
\hline What is your class level? & $\begin{array}{l}\text { Freshman (1) } \\
\text { Sophomore (2) } \\
\text { Junior (3) } \\
\text { Senior (4) } \\
\text { Graduate (5) }\end{array}$ \\
\hline $\begin{array}{l}\text { Are you a transfer student to } \\
\text { Canisius College? }\end{array}$ & $\begin{array}{l}\text { Yes (1) } \\
\text { No (2) }\end{array}$ \\
\hline What is your major? & $\begin{array}{l}\text { Accounting (1) } \\
\text { Accounting Information Systems (2) } \\
\text { Economics (3) } \\
\text { Entrepreneurship (4) } \\
\text { Finance (5) } \\
\text { International Business (6) } \\
\text { Management (7) } \\
\text { Marketing (8) } \\
\text { Other (9) }\end{array}$ \\
\hline $\begin{array}{l}\text { Which program are you pursing } \\
\text { your MBA in: }\end{array}$ & $\begin{array}{l}\text { Flexible (part-time evening ) MBA } \\
\text { One-Year MBA } \\
\text { MBA in Professional Accounting }\end{array}$ \\
\hline $\begin{array}{l}\text { Do you have a concentration? } \\
\text { (MBA) }\end{array}$ & $\begin{array}{l}\text { Yes, I have a concentration. (1) } \\
\text { Undecided on a concentration. (2) } \\
\text { No, I will not be completing a concentration (3) }\end{array}$ \\
\hline $\begin{array}{l}\text { If you are pursuing a concentration, } \\
\text { what concentration are you } \\
\text { pursuing? }\end{array}$ & $\begin{array}{l}\text { Financial Services (1) } \\
\text { Global Supply Chain Management (2) } \\
\text { International Business (3) } \\
\text { Marketing in the New Economy (4) }\end{array}$ \\
\hline Are you: & Female (2) \\
\hline How old are you? & \\
\hline $\begin{array}{l}\text { Would you classify your technology } \\
\text { understanding as: }\end{array}$ & $\begin{array}{l}\text { - Do not use technology. (1) } \\
\text { - Beginner/Novice (e.g. use Microsfot Office } \\
\text { Powerpoint and EXCEl (or similar projection } \\
\text { software) as part of communicating and email). (2) } \\
\text { - Advanced Beginner (e.g. use 'some' Powerpoint (or } \\
\text { similar projection software), some Desire2Learn } \\
\text { features, Microsoft Office or similar to } \\
\text { communicate). (3) }\end{array}$ \\
\hline
\end{tabular}




\begin{tabular}{|l|l|}
\hline & $\begin{array}{l}\text { - Intermediate (e.g. Use Microsoft Office Suite and } \\
\text { other computer applications (such as SPSS, R, etc.) } \\
\text { to communicate and program). (4) } \\
\text { - Extensive (e.g. extensive knowledge and use of } \\
\text { various computer software programs) (5) }\end{array}$ \\
\hline $\begin{array}{l}\text { Have you taken an online course at } \\
\text { Canisius College? }\end{array}$ & $\begin{array}{l}\text { YesCTION A) (1) } \\
\text { No (SECTION B) (2) }\end{array}$ \\
\hline
\end{tabular}

\section{SECTION A - AT LEAST ONE ONLINE COURSE AT CANISIUS COLLEGE}

\begin{tabular}{|c|c|}
\hline How many online courses have you taken: & \\
\hline $\begin{array}{l}\text { Have you taken an online course at a school other than } \\
\text { Canisius College? }\end{array}$ & $\begin{array}{l}\text { Yes }(1) \\
\text { No }(2)\end{array}$ \\
\hline $\begin{array}{l}\text { How many online courses have you taken at a school other } \\
\text { than Canisius College? }\end{array}$ & \\
\hline $\begin{array}{l}\text { Prior to taking an online course, did you take a course to } \\
\text { prepare you for the online environment? }\end{array}$ & Yes (1) \\
\hline $\begin{array}{l}\text { If 'yes', was the course offered by Canisius College or the } \\
\text { book publisher? }\end{array}$ & $\begin{array}{l}\text { Canisius } \\
\begin{array}{l}\text { College (1) } \\
\text { (3) }\end{array}\end{array}$ \\
\hline
\end{tabular}

With respect to the online courses that you have taken at Canisius College compared to face-to-face (traditional classrooms), please rate the following responses:

\begin{tabular}{|c|c|}
\hline $\begin{array}{l}\text { I perceive online courses to be____ in difficulty than face-to- } \\
\text { face courses. }\end{array}$ & $\begin{array}{l}\text { (1) Significantly Easier } \\
\text { (2) Easier } \\
\text { (3) The Same Difficulty } \\
\text { (4) Harder } \\
\text { (5) Significantly Harder }\end{array}$ \\
\hline $\begin{array}{l}\text { I am } \\
\text { courses. }\end{array}$ motivated in online courses than face-to-face & $\begin{array}{l}\text { (1) Significantly Less } \\
\text { (2) Less } \\
\text { (3) Equally } \\
\text { (4) More } \\
\text { (5) Significantly More }\end{array}$ \\
\hline $\begin{array}{l}\text { I the interaction with other students in the online } \\
\text { environment compared to the face-to-face course } \\
\text { environment. }\end{array}$ & $\begin{array}{l}\text { (1) Significantly Dislike } \\
\text { (2) Dislike } \\
\text { (3) Equate } \\
\text { (4) Like } \\
\text { (5) Significantly Like }\end{array}$ \\
\hline $\begin{array}{l}\text { I the interaction with the instructor in the online } \\
\text { environment compared to the face-to-face course } \\
\text { environment. }\end{array}$ & $\begin{array}{l}\text { (1) Significantly Dislike } \\
\text { (2) Dislike } \\
\text { (3) Equate } \\
\text { (4) Like } \\
\text { (5) Significantly Like }\end{array}$ \\
\hline $\begin{array}{l}\text { I find the discipline required in taking online courses to be } \\
\text { than in face-to-face courses. }\end{array}$ & $\begin{array}{l}\text { (1) Significantly Less } \\
\text { (2) Less } \\
\text { (3) The Same } \\
\text { (4) More }\end{array}$ \\
\hline
\end{tabular}




\begin{tabular}{|c|c|}
\hline & (5) Significantly More \\
\hline $\begin{array}{l}\text { I find its' } \quad \text { to cheat in the online environment than in } \\
\text { face-to-face courses. }\end{array}$ & $\begin{array}{l}\text { (1) Significantly Easier } \\
\text { (2) Easier } \\
\text { (3) The Same } \\
\text { (4) Harder } \\
\text { (5) Significantly Harder }\end{array}$ \\
\hline $\begin{array}{l}\text { I enjoy the self-directed online learning environment__than } \\
\text { the interaction in face-to-face courses. }\end{array}$ & $\begin{array}{l}\text { (1) Significantly Less } \\
\text { (2) Less } \\
\text { (3) The Same } \\
\text { (4) More } \\
\text { (5) Significantly More }\end{array}$ \\
\hline $\begin{array}{l}\text { I enjoy the independence associated with the online learning } \\
\text { environment__than the interaction in face-to-face courses. }\end{array}$ & $\begin{array}{l}\text { (1) Significantly Less } \\
\text { (2) Less } \\
\text { (3) The Same } \\
\text { (4) More } \\
\text { (5) Significantly More }\end{array}$ \\
\hline $\begin{array}{l}\text { I enjoy the schedule flexibility associated with the online } \\
\text { learning environment__ than the interaction in face-to-face } \\
\text { courses. }\end{array}$ & $\begin{array}{l}\text { (1) Significantly Less } \\
\text { (2) Less } \\
\text { (3) The Same } \\
\text { (4) More } \\
\text { (5) Significantly More }\end{array}$ \\
\hline $\begin{array}{l}\text { I find online courses require__time investment in the } \\
\text { course than face-to-face courses }\end{array}$ & $\begin{array}{l}\text { (1) Significantly Less } \\
\text { (2) Less } \\
\text { (3) The Same } \\
\text { (4) More } \\
\text { (5) Significantly More }\end{array}$ \\
\hline $\begin{array}{l}\text { I find online courses total costs are ___ than face-to-face } \\
\text { courses. }\end{array}$ & $\begin{array}{l}\text { (1) Significantly Less } \\
\text { (2) Less } \\
\text { (3) The Same } \\
\text { (4) More } \\
\text { (5) Significantly More }\end{array}$ \\
\hline $\begin{array}{l}\text { In the online environment, I feel these activities increase my } \\
\text { understanding of the course material. (Circle all that apply) }\end{array}$ & $\begin{array}{l}\text { (1) Additional Readings (Not } \\
\text { including textbook) } \\
\text { (2) Assignments/Homework } \\
\text { (3) Course Surveys } \\
\text { (4) Discussion boards } \\
\text { (5) In-class sessions (live } \\
\text { sessions) } \\
\text { (6) Instructor chat } \\
\text { (7) Instructor (live or taped) } \\
\text { lectures } \\
\text { (8) Instructor Office Hours } \\
\text { (9) Instructor Posted Notes } \\
\text { (10) Laboratory/ experiential } \\
\text { activities } \\
\text { (11) Other students }\end{array}$ \\
\hline
\end{tabular}




\begin{tabular}{|c|c|}
\hline & $\begin{array}{l}\text { (12) Problem Hints \& Scaffolding } \\
\text { Examples } \\
\text { (13) Textbook } \\
\text { (14) Video of Relevant Course } \\
\text { Material (not instructor-lead) } \\
\text { (15) Other }\end{array}$ \\
\hline $\begin{array}{l}\text { In the online environment, I feel these activities decrease my } \\
\text { understanding of the course material. (Circle all that apply) }\end{array}$ & $\begin{array}{l}\text { (1) Additional Readings (Not } \\
\text { including textbook) } \\
\text { (2) Assignments/Homework } \\
\text { (3) Course Surveys } \\
\text { (4) Discussion boards } \\
\text { (5) In-class sessions (live } \\
\text { sessions) } \\
\text { (6) Instructor chat } \\
\text { (7) Instructor (live or taped) } \\
\text { lectures } \\
\text { (8) Instructor Office Hours } \\
\text { (9) Instructor Posted Notes } \\
\text { (10) Laboratory/ experiential } \\
\text { activities } \\
\text { (11) Other students } \\
\text { (12) Problem Hints \& Scaffolding } \\
\text { Examples } \\
\text { (13) Textbook } \\
\text { (14) Video of Relevant Course } \\
\text { Material (not instructor-lead) } \\
\text { (15) Other }\end{array}$ \\
\hline $\begin{array}{l}\text { Would you prefer to take the class in a traditional face-to-face } \\
\text { environment? }\end{array}$ & Yes (1) Undecided(2) No (3) \\
\hline I am ___ with the online course environment for learning. & $\begin{array}{l}\text { (1) Not very happy } \\
\text { (2) Not happy } \\
\text { (3) Okay } \\
\text { (4) Happy } \\
\text { (5) Very happy }\end{array}$ \\
\hline $\begin{array}{l}\text { Given this institution, do you think online courses are } \\
\text { appropriate? }\end{array}$ & Yes (1) Undecided(2) No (3) \\
\hline Why did you choose to take the course online? & \\
\hline
\end{tabular}

Thank you for your time! It is greatly appreciated. 
SECTION B - NEVER TAKEN AN ONLINE COURSE AT CANISIUS COLLEGE.

\begin{tabular}{|c|c|}
\hline \multicolumn{2}{|c|}{$\begin{array}{l}\text { With respect to the face-to-face (traditional) courses that you have taken at Canisius Colleg } \\
\text { compared to your perception of online courses, please rate the following responses: }\end{array}$} \\
\hline $\begin{array}{l}\text { I perceive face-to-face courses to be __ in difficulty than } \\
\text { online courses. }\end{array}$ & $\begin{array}{l}\text { (1) Significantly Easier } \\
\text { (2) Easier } \\
\text { (3) The Same Difficulty } \\
\text { (4) Harder } \\
\text { (5) Significantly Harder }\end{array}$ \\
\hline $\begin{array}{l}\text { I perceive that I would be ___ motivated in face-to-face } \\
\text { courses than online courses. }\end{array}$ & $\begin{array}{l}\text { (1) Significantly Less } \\
\text { (2) Less } \\
\text { (3) Equally } \\
\text { (4) More } \\
\text { (5) Significantly More }\end{array}$ \\
\hline $\begin{array}{l}\text { I perceive the interaction with other students in the face-to- } \\
\text { face environment to be c_ compared to the online course } \\
\text { environment. }\end{array}$ & $\begin{array}{l}\text { (1) Significantly Worse } \\
\text { (2) Worse } \\
\text { (3) Equal } \\
\text { (4) Better } \\
\text { (5) Significantly Better }\end{array}$ \\
\hline $\begin{array}{l}\text { I perceive the interaction with the instructor and students in } \\
\text { the face-to-face environment to be ___ compared to the } \\
\text { online course environment. }\end{array}$ & $\begin{array}{l}\text { (1) Significantly Worse } \\
\text { (2) Worse } \\
\text { (3) Equal } \\
\text { (4) Better } \\
\text { (5) Significantly Better }\end{array}$ \\
\hline $\begin{array}{l}\text { I perceive the discipline required in taking face-to-face } \\
\text { courses to be__than in online courses. }\end{array}$ & $\begin{array}{l}\text { (1) Significantly Less } \\
\text { (2) Less } \\
\text { (3) The Same } \\
\text { (4) More } \\
\text { (5) Significantly More }\end{array}$ \\
\hline $\begin{array}{l}\text { I perceive that it would be to cheat in the face-to-face } \\
\text { environment than in online courses. }\end{array}$ & $\begin{array}{l}\text { (1) Significantly Easier } \\
\text { (2) Easier } \\
\text { (3) The Same } \\
\text { (4) Harder } \\
\text { (5) Significantly Harder }\end{array}$ \\
\hline $\begin{array}{l}\text { I believe that I would enjoy the self-directed face-to-face } \\
\text { learning environment_than the interaction in online } \\
\text { courses. }\end{array}$ & $\begin{array}{l}\text { (1) Significantly Less } \\
\text { (2) Less } \\
\text { (3) The Same } \\
\text { (4) More } \\
\text { (5) Significantly More }\end{array}$ \\
\hline $\begin{array}{l}\text { I believe that I would enjoy the independence associated with } \\
\text { the face-to-face learning environment___than the interaction } \\
\text { in online courses. }\end{array}$ & $\begin{array}{l}\text { (1) Significantly Less } \\
\text { (2) Less } \\
\text { (3) The Same } \\
\text { (4) More }\end{array}$ \\
\hline
\end{tabular}




\begin{tabular}{|c|c|}
\hline & (5) Significantly More \\
\hline $\begin{array}{l}\text { I believe that I would enjoy the schedule flexibility associated } \\
\text { with the face-to-face learning environment_than in online } \\
\text { courses. }\end{array}$ & $\begin{array}{l}\text { (1) Significantly Less } \\
\text { (2) Less } \\
\text { (3) The Same } \\
\text { (4) More } \\
\text { (5) Significantly More }\end{array}$ \\
\hline $\begin{array}{l}\text { I believe that face-to-face courses require_time } \\
\text { investment in the course than online courses. }\end{array}$ & $\begin{array}{l}\text { (1) Significantly Less } \\
\text { (2) Less } \\
\text { (3) The Same } \\
\text { (4) More } \\
\text { (5) Significantly More }\end{array}$ \\
\hline $\begin{array}{l}\text { I believe face-to-face courses total costs are ___ than online } \\
\text { courses. }\end{array}$ & $\begin{array}{l}\text { (1) Significantly Less } \\
\text { (2) Less } \\
\text { (3) The Same } \\
\text { (4) More } \\
\text { (5) Significantly More }\end{array}$ \\
\hline $\begin{array}{l}\text { In the face-to-face environment, I feel these activities increase } \\
\text { my understanding of the course material. (Circle all that } \\
\text { apply) }\end{array}$ & $\begin{array}{l}\text { (1) Additional Readings (Not } \\
\text { including textbook) } \\
\text { (2) Assignments/Homework } \\
\text { (3) Course Surveys } \\
\text { (4) Discussion boards } \\
\text { (5) In-class sessions (live } \\
\text { sessions) } \\
\text { (6) Instructor chat } \\
\text { (7) Instructor (live or taped) } \\
\text { lectures } \\
\text { (8) Instructor Office Hours } \\
\text { (9) Instructor Posted Notes } \\
\text { (10) Laboratory/ experiential } \\
\text { activities } \\
\text { (11) Other students } \\
\text { (12) Problem Hints \& Scaffolding } \\
\text { Examples } \\
\text { (13) Textbook } \\
\text { (14) Video of Relevant Course } \\
\text { Material (not instructor-lead) } \\
\text { (15) Other }\end{array}$ \\
\hline $\begin{array}{l}\text { In the online environment, I feel these activities decrease my } \\
\text { understanding of the course material. (Circle all that apply) }\end{array}$ & $\begin{array}{l}\text { (1) Additional Readings (Not } \\
\text { including textbook) } \\
\text { (2) Assignments/Homework } \\
\text { (3) Course Surveys } \\
\text { (4) Discussion boards } \\
\text { (5) In-class sessions (live } \\
\text { sessions) } \\
\text { (6) Instructor chat }\end{array}$ \\
\hline
\end{tabular}




\begin{tabular}{|c|c|}
\hline & $\begin{array}{l}\text { (7) Instructor (live or taped) } \\
\text { lectures } \\
\text { (8) Instructor Office Hours } \\
\text { (9) Instructor Posted Notes } \\
\text { (10) Laboratory/ experiential } \\
\text { activities } \\
\text { (11) Other students } \\
\text { (12) Problem Hints \& Scaffolding } \\
\text { Examples } \\
\text { (13) Textbook } \\
\text { (14) Video of Relevant Course } \\
\text { Material (not instructor-lead) } \\
\text { (15) Other }\end{array}$ \\
\hline Would you prefer to take the class in an online environment? & Yes (1) Undecided(2) No (3) \\
\hline \multicolumn{2}{|l|}{ If 'no', why not? } \\
\hline I am ___ with the face-to-face environment for learning. & $\begin{array}{l}\text { (1) Not very happy } \\
\text { (2) Not happy } \\
\text { (3) Okay } \\
\text { (4) Happy } \\
\text { (5) Very happy }\end{array}$ \\
\hline $\begin{array}{l}\text { Given this institution, do you think online courses are } \\
\text { appropriate? }\end{array}$ & Yes (1) Undecided(2) No (3) \\
\hline $\begin{array}{l}\text { Have you taken an online course at a school other than } \\
\text { Canisius College? }\end{array}$ & $\begin{array}{l}\text { Yes (1) } \\
\text { No (2) }\end{array}$ \\
\hline
\end{tabular}

Thank you for your time! It is greatly appreciated. 